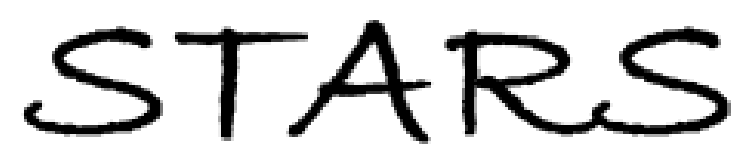

University of Central Florida

STARS

Faculty Bibliography 2000s

Faculty Bibliography

$1-1-2007$

\title{
Anchoring energy and cell gap effects on liquid crystal response time
}

Xiangyi Nie

University of Central Florida

Ruibo Lu

University of Central Florida

Haiqing Xianyu

University of Central Florida

Thomas X. Wu

University of Central Florida

Shin-Tson Wu

University of Central Florida

Find similar works at: https://stars.library.ucf.edu/facultybib2000

University of Central Florida Libraries http://library.ucf.edu

This Article is brought to you for free and open access by the Faculty Bibliography at STARS. It has been accepted for inclusion in Faculty Bibliography 2000s by an authorized administrator of STARS. For more information, please contact STARS@ucf.edu.

\section{Recommended Citation}

Nie, Xiangyi; Lu, Ruibo; Xianyu, Haiqing; Wu, Thomas X.; and Wu, Shin-Tson, "Anchoring energy and cell gap effects on liquid crystal response time" (2007). Faculty Bibliography 2000s. 7470.

https://stars.library.ucf.edu/facultybib2000/7470

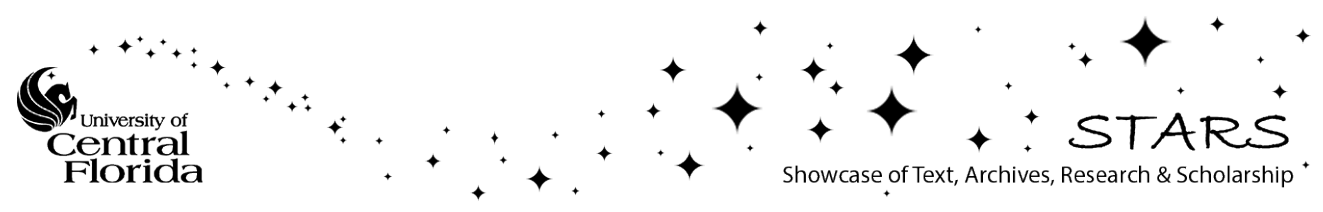




\section{Anchoring energy and cell gap effects on liquid crystal response time}

Cite as: J. Appl. Phys. 101, 103110 (2007); https://doi.org/10.1063/1.2734870

Submitted: 16 February 2007 . Accepted: 17 March 2007 . Published Online: 25 May 2007

Xiangyi Nie, Ruibo Lu, Haiqing Xianyu, Thomas X. Wu, and Shin-Tson Wu

\section{ARTICLES YOU MAY BE INTERESTED IN}

Anchoring energy enhancement and pretilt angle control of liquid crystal alignment on polymerized surfaces

AIP Advances 5, 097218 (2015); https://doi.org/10.1063/1.4932153

Alignment layer effects on thin liquid crystal cells

Applied Physics Letters 92, 061102 (2008); https://doi.org/10.1063/1.2841642

A novel method for determining the anchoring energy function at a nematic liquid crystal-wall interface from director distortions at high fields

Journal of Applied Physics 57, 4520 (1985); https://doi.org/10.1063/1.335352

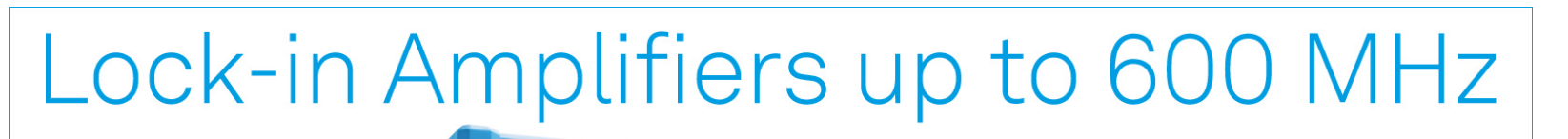

starting at

$$
\$ 6,210
$$

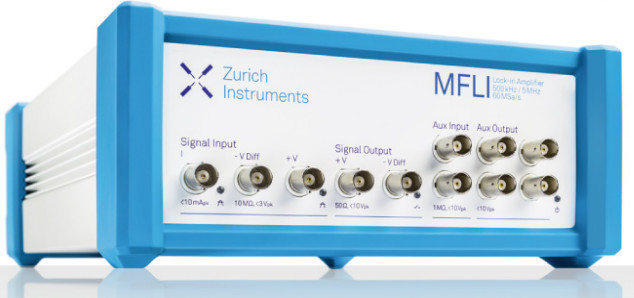

J. Appl. Phys. 101, 103110 (2007); https://doi.org/10.1063/1.2734870

(c) 2007 American Institute of Physics.

101, 103110 


\title{
Anchoring energy and cell gap effects on liquid crystal response time
}

\author{
Xiangyi Nie, Ruibo Lu, Haiqing Xianyu, Thomas X. Wu, and Shin-Tson Wu ${ }^{a}$ \\ College of Optics and Photonics, University of Central Florida, Orlando, Florida 32816
}

(Received 16 February 2007; accepted 17 March 2007; published online 25 May 2007)

\begin{abstract}
The anchoring energy and cell gap effects on liquid crystal response time $\left(\tau_{0}\right)$ is analyzed theoretically and validated experimentally. Analytical expressions are derived using two different approaches: effective cell gap and surface dynamic equation methods. Consistent results are deduced from these two approaches. A simplified equation $\tau_{0} \sim d^{x}$ also fits the experimental data well, where $d$ is the liquid crystal cell gap and $x$ is the exponent. Under two extreme (strong and weak) anchoring limits, the exponent $x$ approaches 2 and 1, respectively. This information is helpful for optimizing liquid crystal devices for display applications. (C) 2007 American Institute of Physics.
\end{abstract} [DOI: $10.1063 / 1.2734870]$

\section{INTRODUCTION}

The response time of a liquid crystal (LC) plays a crucial role for LC devices. The conventional understanding is that LC response (decay) time $\tau_{0}$ is proportional to $d^{2}$, where $d$ is the cell gap. ${ }^{1}$ Here, the underlining assumption is that the anchoring energy on LC-substrate surfaces is strong $(W \rightarrow \infty)$. However, some LC devices have relatively weak anchoring energy, such as LC cells with multidomain vertical alignment (MVA) or with photoalignment. As a result, the $\tau_{0} \sim d^{2}$ dependence no longer holds. Therefore, it is essential to study the anchoring energy and cell gap effects on the LC response time.

In this paper, we use two different approaches: effective cell gap and surface dynamic equation methods, to derive general analytical expressions of LC response time under finite anchoring energy conditions. The results of these two different approaches are consistent. We find that the exponent $x$ in $\tau_{0} \sim d^{x}$ depends on the anchoring energy $W$ of the LC cell. Under strong and weak anchoring limits, the exponent $x$ approaches 2 and 1, respectively.

In this paper, the derived analytical expressions correlate LC response time $\tau_{0}$ with anchoring energy $W$, then $W$ can be estimated from LC decay time. Up to date, a few experimental methods have been developed for characterizing the anchoring energy of LC cells. ${ }^{2-5}$ Most of them are based on the anchoring energy effect on LC cell's phase retardation in the high voltage region or its effect on the Freekdericksz transition. The method proposed in this paper provides another approach to study LC alignment techniques.

\section{THEORY}

\section{A. Effective cell gap method}

In this study, we choose a vertically aligned (VA) LC cell as an example for analysis because it exhibits an unprecedented contrast ratio (>2000:1) and more than 50\% of liquid crystal display televisions (LCD TVs) use VA mode. These results and discussions are also valid to other LC modes. Figure 1 shows a VA nematic LC cell sandwiched

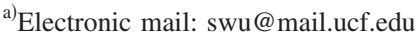

between two parallel substrates and $z=-d / 2$ and $d / 2$ represent the bottom and top substrates, respectively. The $z$ axis is normal to the plane of the substrates and the electrical field $E$ is along the $z$ axis.

Under such a device configuration, the LC dynamics can be described by the Erickson-Leslie equation. When the backflow and inertial effects are ignored, ${ }^{6,7}$ the EricksonLeslie equation has the following simplified expression:

$$
\begin{aligned}
& \left(K_{11} \sin ^{2} \theta+K_{33} \cos ^{2} \theta\right) \frac{\partial^{2} \theta}{\partial Z^{2}} \\
& \quad+\left(K_{33}-K_{11}\right) \sin \theta \cos \theta\left(\frac{\partial \theta}{\partial Z}\right)^{2}+\varepsilon_{0} \Delta \varepsilon E^{2} \sin \theta \cos \theta \\
& =-\gamma_{1} \frac{\partial \theta}{\partial t},
\end{aligned}
$$

where $\gamma_{1}$ is the rotational viscosity, $K_{11}$ and $K_{33}$ represent the splay and bend elastic constants, respectively, $\varepsilon_{0} \Delta \varepsilon E^{2}$ is the electric field energy density, $\Delta \varepsilon$ is the LC dielectric anisotropy, and $\theta$ is the tilt angle defined as the angle between the $z$ axis and LC directors. In general, Eq. (1) can only be solved numerically. However, when the tilt angle is small $(\sin \theta \sim \theta) \quad\left(\right.$ small angle approximation $\left.{ }^{8}\right)$ and $K_{33} \sim K_{11}$ (single elastic constant approximation), the Erickson-Leslie equation is reduced to

$$
K_{33} \frac{d^{2} \theta}{d z^{2}}+\varepsilon_{0} \Delta \varepsilon E^{2} \theta=-\gamma_{1} \frac{\partial \theta}{\partial t} .
$$

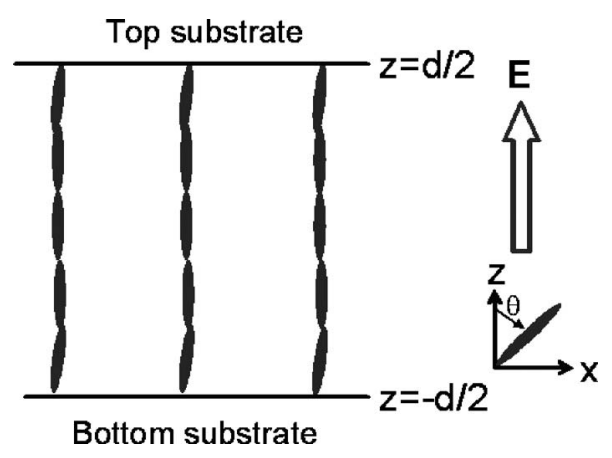

FIG. 1. Schematic drawing of a VA LC cell. 


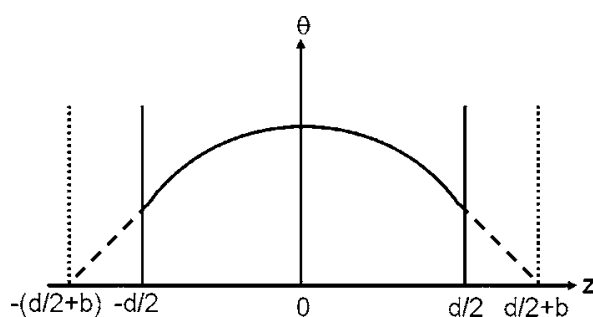

FIG. 2. The z-coordinate dependent tilt angle $\theta$. The LC cell's physical surfaces are at $z= \pm d / 2$. The $\theta$ profile is extrapolated to $z= \pm(d / 2+b)$, where $\theta=0 . b=K / W$ is the extrapolation length.

Under strong anchoring $(W \rightarrow \infty)$ condition, the LC directors on the substrates are fixed on their easy axis. Here, easy axis stands for the directions of LC directors which minimize the energy of the surface regions. ${ }^{9}$ In some high contrast display devices, the pretilt angle is zero. Under such condition, the following boundary conditions hold:

$$
\theta_{Z=-d / 2, d / 2}=0 \text {. }
$$

Under the above boundary conditions, when the applied voltage exceeds the Freederisckz transition threshold the LC directors are reoriented by the electric field. At a given voltage, the tilt angle can be expressed as follows:

$$
\theta=\left[\theta_{s} \sin (\beta z)+\theta_{m} \cos (\beta z)\right] \exp (-t / \tau) .
$$

In Eq. (4), $\theta_{m}$ is the maximum tilt angle which occurs in the center of the LC cell $\left(\left.\theta\right|_{z=0}=\theta_{m}\right)$, and $\theta_{s}$ is found to be zero when the pretilt angle is symmetric on the top and bottom substrates. Under these conditions, analytical solutions for rise time $\left(\tau_{r}\right)$ and decay time $\left(\tau_{d}\right)$ exist: $^{8}$

$$
\begin{aligned}
& \tau_{d}=\tau_{0}=\frac{\gamma_{1} d^{2}}{K_{33} \pi^{2}}, \\
& \tau_{r}=\frac{\gamma_{1}}{\left|\varepsilon_{0}\right| \Delta \varepsilon\left|E^{2}-\left(\pi^{2} / d^{2}\right) K_{33}\right|}=\frac{\tau_{0}}{\left|\left(V / V_{\mathrm{th}}\right)^{2}-1\right|} .
\end{aligned}
$$

In Eq. (6), the threshold voltage $V_{\text {th }}$ is related to the bend elastic constant and dielectric anisotropy as

$$
V_{\mathrm{th}}=\pi \sqrt{\frac{K_{33}}{\varepsilon_{0}|\Delta \varepsilon|}} .
$$

However, when the anchoring energy of the LC cell is not strong, the simple boundary conditions described in Eq. (3) no longer hold, and the validity of Eqs. (5) and (6) is in doubt. When the anchoring energy $W$ is finite, we use the extrapolation length concept ${ }^{9}$ to derive the analytical solutions for the LC response time.

As shown in Fig. 2, the parameter $b=K / W$ has the dimension of length, which is usually called the extrapolation length and $W$ is the polar anchoring energy strength coefficient, also called as the anchoring energy. For a VA cell, the azimuthal anchoring is not involved and $K=K_{33}$, the bend elastic constant.

The extrapolation length $b$ can be interpreted as the extension of the LC cell gap. ${ }^{10}$ For example, an infinity anchoring $(W \rightarrow \infty)$ means no extension since $b=0$. On the other hand, weak anchoring implies to a large $b$, i.e., a large ex- tension of the LC cell gap. For a LC cell with a finite anchoring energy, the LC directors on the physical substrate boundaries $(z= \pm d / 2)$ will still be reoriented by the external electric fields. According to the definition of the extrapolation length, $\theta$ is zero at $z= \pm(d / 2+b)$. Therefore, $z= \pm(d / 2$ $+b)$ can be considered as virtual boundaries of the LC cell and the effective cell gap becomes

$$
d^{\prime}=d+2 b
$$

Here we consider the top and bottom substrates having the same alignment conditions. If the two substrates' alignments are different, then the effective cell gap is $d^{\prime}=d+b_{b}$ $+b_{t}$, where $b_{b}$ and $b_{t}$ represent the extrapolation length of the bottom and top substrates, respectively.

Based on this effective cell gap concept, we can modify Eqs. (5) and (6) to further derive the LC response time when $W$ is finite. Both decay time $\tau_{0}^{\prime}$ and rise time $\tau_{r}^{\prime}$ can be derived if we replace the physical cell gap $d$ with the effective cell gap $d^{\prime}$,

$$
\tau_{0}^{\prime}=\frac{\gamma_{1} d^{\prime 2}}{K \pi^{2}}=\frac{\gamma_{1}}{K \pi^{2}}(d+2 b)^{2}=\frac{\gamma_{1}}{K \pi^{2}}\left(d^{2}+\frac{4 d K}{W}+\frac{4 K^{2}}{W^{2}}\right) .
$$

From Eq. (9), if $2 b \ll d$, then the anchoring effect is negligible and $d^{\prime} \approx d$. Under the strong anchoring limit, Eq. (9) is reduced to Eq. (5). For an intermediate anchoring strength, $2 K / W$ is not completely negligible. Therefore, we have to consider the anchoring energy terms shown in the right hand of Eq. (9). Because the quadratic term $\left[(K / W)^{2}\right]$ in Eq. (9) is independent of the cell gap and its magnitude is small as will be shown later, for the interest of understanding the cell gap effect, we only consider the first two terms. Under this condition, Eq. (9) reads as

$$
\tau_{0}^{\prime} \approx \frac{\gamma_{1}}{K \pi^{2}}\left(d^{2}+\frac{4 d K}{W}\right)
$$

This equation suggests that $\tau_{0}^{\prime} \sim d^{2}$ is accurate if $d \gg 4 K / W$, i.e., strong anchoring energy, but when $W$ is small, the firstorder term $4 d K / W$ has to be considered and $\tau_{0}^{\prime} \sim d^{2}$ is no longer accurate. Under a very weak anchoring condition where $4 K / W \gg d$, the LC directors response time is reduced to

$$
\tau_{0}^{\prime} \approx \frac{4 \gamma_{1} d}{W \pi^{2}}
$$

Equation (10) suggests that the exponent $x$ in $\tau_{0}^{\prime} \sim d^{x}$ lies between 1 and 2, where $x \sim 1$ if the anchoring energy is very weak while $x \sim 2$ if the anchoring energy is very strong.

Similar correlations hold for rise time $\tau_{r}^{\prime}$ as well, i.e., by replacing $d$ with $d^{\prime}$ in Eq. (6). Please note that threshold voltage $V_{\text {th }}$ is also dependent on the anchoring energy. ${ }^{5}$ Thus, Eq. (7) needs to be modified when $W$ has a finite value. As a result, Eq. (6) would have a much more complicated expression. 


\section{B. Surface dynamic equation method}

Another approach to study the anchoring energy effect is to use the surface dynamic equation method. ${ }^{11}$ If the anchoring energy is finite, the simple boundary condition described in Eq. (3) is no longer valid. Instead, the surface dynamic equation can be used to represent LC directors' reorientation on the LC-substrate boundaries,

$$
\left(K_{11} \sin ^{2} \theta+K_{33} \cos ^{2} \theta\right) \frac{\partial \theta}{\partial Z} \pm \frac{d f_{s}}{d \theta}=\gamma_{s} \frac{\partial \theta}{\partial t},
$$

where, $f_{s}=\frac{1}{2} W \sin ^{2} \theta$ stands for the anchoring energy density on the boundaries, and $\gamma_{s}$ is the surface rotational viscosity, and "+" and "-" signs are taken at $z=d / 2$ and $z=-d / 2$, respectively. Analytical expressions can be derived following the approach reported in Ref. 11. Because the characteristic time of the surface LC director reorientation is much longer than that of the corresponding LC directors in the bulk, the surface term $\gamma_{s} \partial \theta / \partial t$ in Eq. (12) can be ignored. Under small angle approximation, Eq. (12) is simplified to

$$
K_{33} \frac{\partial \theta}{\partial Z} \pm W \theta=0
$$

By solving Eqs. (4) and (13), we derive the following decay time and rise time:

$$
\begin{aligned}
& \tau_{d}^{\prime}=\tau_{0}^{\prime}=\frac{\gamma_{1}}{\beta^{2} K_{33}}, \\
& \tau_{r}^{\prime}=\frac{\gamma_{1}}{\left|\varepsilon_{0}\right| \Delta \varepsilon\left|E^{2}-\beta^{2} K_{33}\right|},
\end{aligned}
$$

where $\beta$ satisfies the following equation:

$$
\operatorname{tg}(\beta d)=\frac{2 W / K_{33} \beta}{1-\left(W / K_{33} \beta\right)^{2}} .
$$

If $W \rightarrow \infty$ (strong anchoring), the right term of Eq. (16) approaches zero from the negative side. Thus, we find $\beta$ $=\pi / d$. Under this condition, Eqs. (14) and (15) are reduced to Eqs. (5) and (6).

If $W \rightarrow 0, \operatorname{tg}(\beta d)$ is small and it can be approximated by $\beta d$. Solving Eq. (16) we obtain

$$
\beta^{2} \approx \frac{\left(W d / K_{33}+2\right) W}{K_{33} d} .
$$

When $W d / K_{33} \ll 1$, Eq. (17) can be approximated as $\beta^{2}$ $\approx 2 W /\left(K_{33} d\right)$. Under such a circumstance, the decay time and rise time have following expressions:

$$
\begin{aligned}
& \tau_{d}^{\prime}=\tau_{0}^{\prime} \approx \frac{\gamma_{1} d}{2 W}, \\
& \tau_{r}^{\prime}=\frac{\gamma_{1}}{\left|\varepsilon_{0}\right| \Delta \varepsilon\left|E^{2}-2 W / d\right|} .
\end{aligned}
$$

Equation (18) also leads to $\tau_{0}^{\prime} \sim d$ for the case of the weak anchoring energy, which is consistent with the result [Eq. (11)] we derived using the effective cell gap method. The only difference is their coefficients. In Eq. (11), the coefficient is $(2 / \pi)^{2}$ which is $\sim 0.41$, while in Eq. (18) the coeffi-

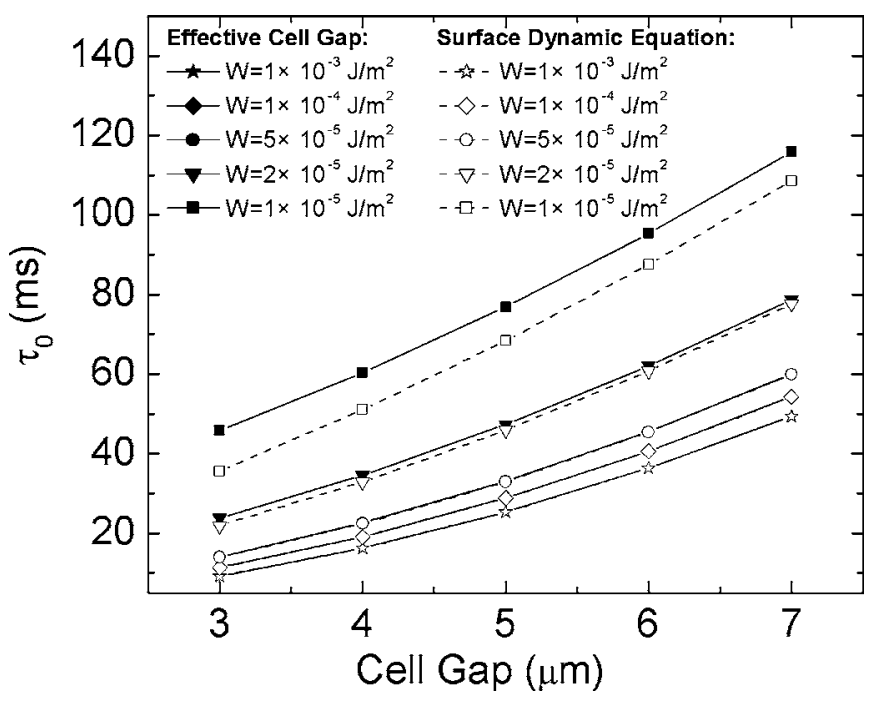

FIG. 3. Anchoring energy $(W)$ and cell gap $(d)$ dependent LC decay time $\left(\tau_{d}^{\prime}=\tau_{0}^{\prime}\right)$. The solid lines represent the results of the effective cell gap method, and the dashed lines are based on the surface dynamic equation method. When $W$ is large, the results of these two methods are almost identical as the bottom three curves $\left(W=1 \times 10^{-3}, 1 \times 10^{-4}\right.$, and 5 $\times 10^{-5} \mathrm{~J} / \mathrm{m}^{2}$ ) show.

cient is 0.5 . This $20 \%$ difference arises from the approximations during the derivation processes of Eqs. (11) and (18).

\section{Results and discussion}

For a LC device, the total response time is usually referred to the sum of rise and decay times. The rise time is strongly dependent on the applied voltage, as shown in Eq. (6). When the applied voltage is only slightly above $V_{\text {th }}$, the rise time is slow. To overcome the slow rise time, an overdrive voltage technique ${ }^{12}$ has been commonly practiced in LCD industry. Therefore, in this paper we focus our discussion on the LC decay process.

Figure 3 shows the simulated cell gap dependent LC decay time under various anchoring strengths. The LC mixture used for calculations is Merck MLC-6608 whose physical properties (at $20{ }^{\circ} \mathrm{C}$ ) are listed as follows: $K_{11}$ $=16.7 \mathrm{pN}, K_{33}=18.1 \mathrm{pN}, \varepsilon_{\|}=3.6, \varepsilon_{\perp}=7.8, \gamma_{1}=0.186 \mathrm{~Pa}, n_{0}$ $=1.475$, and $n_{e}=1.558$. Five cell gaps were studied: $d=3,4$, 5,6 , and $7 \mu \mathrm{m}$. As the cell gap increases, the first transmission maximum occurs at a lower voltage, but the threshold voltage remains the same. In each cell, the assumption of the small angle approximation is still valid.

During simulations, we calculate the LC director decay time from the first transmission maximum to $V=0$. We then use the extrapolation length method (solid lines) and surface dynamic equation method (dashed lines) to fit the simulated data using $\tau_{0} \sim d^{x}$. Table I lists the obtained $x$ values from the solid lines fittings. From Table I, we find that $x \approx 2$ is valid only when the anchoring is strong $\left(W \sim 1 \times 10^{-3} \mathrm{~J} / \mathrm{m}^{2}\right)$. As the anchoring energy gets weaker, the exponent gets closer to 1. These results confirm that the conventional understanding of $\tau_{0} \sim d^{2}$ is only valid when $W \rightarrow \infty$, and the quadratic dependence is no longer accurate if the LC cells have a finite anchoring energy. 
TABLE I. Anchoring energy $W$ dependent $x$ value where $x$ is the exponent of $\tau_{0} \sim d^{x}$. The data are obtained from the fitting of solid lines in Fig. 3.

\begin{tabular}{cccccc}
\hline \hline$W\left(\mathrm{~J} / \mathrm{m}^{2}\right)$ & $1 \times 10^{-3}$ & $1 \times 10^{-4}$ & $5 \times 10^{-5}$ & $2 \times 10^{-5}$ & $1 \times 10^{-5}$ \\
\hline$x$ & 1.98 & 1.84 & 1.71 & 1.41 & 1.09 \\
\hline \hline
\end{tabular}

In Fig. 3, the effective cell gap and surface dynamic equation methods lead to consistent results when the anchoring energy is larger than $1 \times 10^{-4} \mathrm{~J} / \mathrm{m}^{2}$. However, an appreciable amount of discrepancy $(\sim 30 \%)$ is observed when the anchoring energy is low $\left(W=1 \times 10^{-5} \mathrm{~J} / \mathrm{m}^{2}\right)$. We will discuss its causes later. The surface dynamic equation method is relatively complicated, since a simple analytical solution of Eq. (16) is not available. Therefore, we have to solve Eq. (16) numerically. This is especially difficult when $\beta d \sim \pi / 2$ in Eq. (16). If the alignment conditions are different on the bottom and top substrates, then the surface dynamic equation method would be even more complicated. Since these two methods show similar results in the major cases shown in Fig. 3, the effective cell gap method is easier and more practical.

To further analyze the accuracy of the effective cell gap method, two different extrapolation length conditions are plotted in Fig. 4. In Fig. 4(a), $d \gg b$ and the extrapolation length method is pretty accurate. But when the cell gap is relatively small and the anchoring energy is weak as Fig. 4(b) shows, the extrapolation length method would be less accurate. In Fig. 3, if we compare the two curves for $W=1$ $\times 10^{-5} \mathrm{~J} / \mathrm{m}^{2}$, the difference between the solid and dashed lines is attributed by this approximation. When $W=1$ $\times 10^{-5} \mathrm{~J} / \mathrm{m}^{2}$, the $b(=K / W)$ value is as large as $1.81 \mu \mathrm{m}$. Thus, the linear extrapolation of the LC cell gap would not be accurate for thin cells. For instance, if $d=3 \mu \mathrm{m}$ the difference between these two methods is $28.7 \%$, but for a $7 \mu \mathrm{m}$ LC cell, the difference is reduced to $7.4 \%$.

\section{EXPERIMENT}

Based on Eq. (8), we can estimate anchoring energy $W$ by measuring the LC decay time. Since LC decay time is related to the LC material's properties $\left(\gamma_{1}\right.$ and $\left.K\right)$, cell gap $d$, and anchoring energy $W$, we can calculate $W$ by measuring $\tau_{0}$, provided that $\gamma_{1}, K$, and $d$ are known. From Fig. 3, we find that the LC decay time is especially sensitive to the anchoring energy when the anchoring energy is in the intermediate to weak range. Therefore, this method is applicable to LC cells with a relatively weak anchoring energy.

Two types of VA cells have been used for display applications: strong rubbing and rubbing-free. ${ }^{1}$ The rubbed or

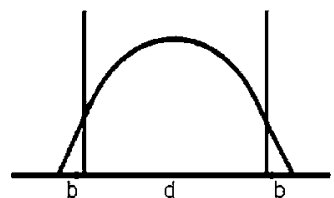

(a)

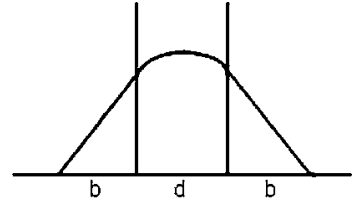

(b)
FIG. 4. (a) When $d \gg b$, the extrapolation length approximation is accurate; (b) when $d \sim b$, the approximation is less accurate. sputtered cells tend to have strong anchoring energy and the rubbing-free cells tend to have a weak anchoring energy. In the former case, such as liquid crystal on silicon for projection displays, a uniform pretilt angle $\left(\sim 2^{\circ}-3^{\circ}\right)$ is generated by sputtered inorganic $\mathrm{SiO}_{2}$ layers. ${ }^{13}$ In the wide-view LCD TVs, patterned vertical alignment ${ }^{14}$ (PVA) and MVA ${ }^{15}$ cells are the two common choices. In these cells, both top and bottom substrates are coated with a thin polyimide layer but without rubbing. In a PVA cell, there is no pretilt angle; the LC directors' reorientation direction is controlled by the fringing fields. In a MVA cell, a small pretilt angle exists only near the protrusions.

In our experiment, we used MVA cells with various cell gaps. The cell gap was measured by counting the FabryPerot interference fringes from a spectrophotometer. The cells were filled with a commercial LC mixture MLC-6608. We first measured the voltage-dependent transmittance between crossed polarizers and then measured the optical decay time. All the measurements were performed using a $\mathrm{He}-\mathrm{Ne}$ laser beam. Its wavelength is $\lambda=633 \mathrm{~nm}$.

Figure 5 shows the measured LC optical decay time $(90 \%-10 \%)^{16}$ of several MVA cells. The experimental data (dots) were collected at $38.5^{\circ} \mathrm{C}$, which is the LCD TV's operating temperature after being fully warmed up. We then fit the data using three models: The solid line stands for the effective cell gap model [Eq. (9)] with $W=5.6 \times 10^{-5} \mathrm{~J} / \mathrm{m}^{2}$, dashed lines for the surface dynamic equation [Eq. (18)] with $W=5.6 \times 10^{-5} \mathrm{~J} / \mathrm{m}^{2}$, and dotted lines for the simplified equation $\tau_{0} \sim d^{x}$ with $x=1.7$. These three curves all fit the experimental data well. When $W=5.6 \times 10^{-5} \mathrm{~J} / \mathrm{m}^{2}$, the extrapolation length of these cells is calculated to be $b=0.34 \mu \mathrm{m}$. For example, if a LC cell has a $3.5 \mu \mathrm{m}$ cell gap, then the $d^{2}$,

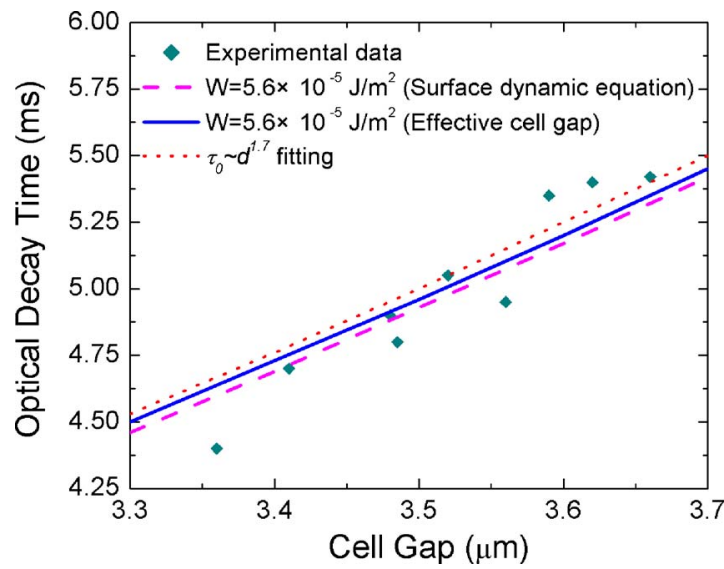

FIG. 5. (Color online) Experimental results of the cell gap dependent LC optical decay time. Stars are the measurement data. The solid line is the fitting curve using the effective cell gap method [Eq. (9)], and the pink dashed line is based on the surface dynamic equation method. From fittings, the anchoring energy is found to be $W=5.6 \times 10^{-5} \mathrm{~J} / \mathrm{m}^{2}$. The red dotted lines represent the fitting curve using $\tau_{0} \sim d^{1.7}$. 
$4 d K / W$, and $4 K^{2} / W^{2}$ terms in Eq. (9) contribute $70.3 \%$, $26.1 \%$, and $2.6 \%$ to $\tau_{0}^{\prime}$, respectively. This confirms that in Eq. (10) the $4 d K / W$ term needs to be considered, but the $4 K^{2} / W^{2}$ term is negligible.

It is known that the LC material and cell gap significantly influence LC device's response time, but the anchoring effect is not well studied quantitatively previously. From Eqs. (11) and (18), the anchoring energy plays an important role to affect the LC response time. Within the weak anchoring regime, the LC decay time is inversely proportional to the anchoring energy. For instance, if we can find a polyimide that has a somewhat larger anchoring with the LC material, then the decay time can be improved.

\section{CONCLUSION}

We derived the analytical expressions for understanding the anchoring energy effect on the LC response time. Two different approaches, namely, extrapolation length and surface dynamics, are employed to study this effect. Both models fit with experimental data well. In addition, a simplified equation $\tau_{0} \sim d^{x}$ was used to fit the experimental data. Under strong and weak anchoring limits, the exponent is close to $x \sim 2$ and $x \sim 1$, respectively. For the tested MVA cells, the anchoring energy is finite and the exponent is found to be $x \sim 1.7$. By optimizing the LC and polyimide interactions, it is possible to optimize the $\mathrm{LC}$ response time.

\section{ACKNOWLEDGMENTS}

The authors are indebted to Chi-Mei Optoelectronics Corporation for financial supports and Dr. C. K. Wei for providing the MVA test cells.

${ }^{1}$ S. T. Wu and D. K. Yang, Reflective Liquid Crystal Displays (Wiley, New York, 2001).

${ }^{2}$ H. Yokoyama and H. A. van Sprang, J. Appl. Phys. 57, 4520 (1985).

${ }^{3}$ Yu. A. Nastishin, R. D. Polak, S. V. Shiyanovskii, and O. D. Lavrentovich, Appl. Phys. Lett. 75, 202 (1999).

${ }^{4}$ X. Nie, Y. H. Lin, T. X. Wu, H. Wang, Z. Ge, and S. T. Wu, J. Appl. Phys. 98, 013516 (2005).

${ }^{5}$ K. H. Yang and C. Rosenblatt, Appl. Phys. Lett. 43, 62 (1983).

${ }^{6}$ J. L. Erickson, Trans. Soc. Rheol. 5, 23 (1961).

${ }^{7}$ F. M. Leslie, Arch. Ration. Mech. Anal. 28, 265 (1968).

${ }^{8}$ E. Jakeman and E. P. Raynes, Phys. Lett. 39A, 69 (1972).

${ }^{9}$ P. G. de Gennes and J. Prost, The Physics of Liquid Crystals (Clarendon Press, Oxford, 1993).

${ }^{10}$ A. A. Sonin, The Surface Physics of Liquid Crystals (Gordon and Breach, New York, 1995).

${ }^{11}$ L. M. Blinov and V. G. Chigrinov, Electrooptic Effects in Liquid Crystal Materials, (Springer-Verlag, Berlin, 1994).

${ }^{12}$ S. T. Wu and C. S. Wu, J. Appl. Phys. 65, 527 (1989).

${ }^{13} \mathrm{D}$. Armitage, I. Underwood, and S. T. Wu, Introduction to Microdisplays (Wiley, New York, 2006).

${ }^{14}$ J. O. Kwag, K. C. Shin, J. S. Kim, S. G. Kim, and S. S. Kim, SID Tech. Digest 31, 256 (2000).

${ }^{15}$ A. Takeda, S. Kataoka, T. Sasaki, H. Chida, K. Ohmuro, and Y. Koike, SID Tech. Digest 29, 1077 (1998).

${ }^{16}$ H. Wang, T. X. Wu, X. Zhu, and S. T. Wu, J. Appl. Phys. 95, 5502 (2004). 